\title{
ADOÇÃO DE PRÁTICAS DE GESTÃ̃ VERDE DA CADEIA DE VALOR EM PMES DO SETOR QUÍMICO: ESTUDOS DE CASO MÚLTIPLOS
}

\section{ADOPTION OF GREEN VALUE CHAIN MANAGEMENT PRACTICES IN CHEMICAL SME: MULTIPLE CASE STUDIES}

\author{
Andréia Fernandes Malaquias ${ }^{1}$; Luis Antonio De Santa-Eulalia ${ }^{2}$; Rui Tadashi Yoshino ${ }^{3}$; David \\ Calchau Jorge ${ }^{4}$ \\ ${ }^{1}$ Universidade Federal do Triângulo Mineiro - MG - Brasil \\ andreiafmalaquias@yahoo.com.br \\ ${ }^{2}$ Université de Sherbrooke - Canadá \\ 1.santa-eulalia@usherbrooke.ca \\ ${ }^{3}$ Universidade Tecnológica Federal do Paraná - PR - Brasil \\ ruiyoshino@utfpr.edu.br \\ ${ }^{4}$ Universidade Federal do Triângulo Mineiro - MG - Brasil \\ david@eletrica.uftm.edu.br
}

\begin{abstract}
Resumo
Devido a sua relevância para a atualidade, a sustentabilidade ambiental é um tema que tem entrado na agenda de muitas empresas modernas. Como parte deste fenômeno, pesquisas recentes têm demonstrado que a abordagem conhecida como "gestão verde da cadeia de valor" tem potencial para contribuir para reduzir o impacto ambiental das empresas. No entanto, boa parte dos estudos empíricos visa grandes empresas, sendo que pouco se sabe sobre como as Pequenas e Médias Empresas (PME) tem adotado tais práticas. Além do mais, não há indícios de como as PMEs do Triângulo Mineiro, particularmente as empresas químicas de Uberaba, tem se modernizado no sentido de incorporar práticas recentes de gestão sustentável. Essa região possui um parque industrial importante contando com diversas empresas do setor químico, sendo que este tipo de empresa é comumente associado à degradação ambiental. Assim, o objetivo deste trabalho é analisar, por meio de uma série de dez estudos de caso, o processo de adoção de práticas sustentáveis em PME do setor químico em Uberaba. Para tanto, levanta-se os principais motivadores de adoção, os tipos de práticas sendo adotadas e os possíveis impactos econômicos e ambientais percebidos pelos gestores destas empresas. Os resultados deste estudo evidenciam uma associação positiva entre as práticas de gestão verde de cadeia de valor com o desempenho econômico e ambiental das empresas estudadas. Além disso, evidencia-se os resultados obtidos pelas PMEs são devidos a motivação pessoal dos empreendedores e líderes das empresas.
\end{abstract}

Palavras-chave: gestão da cadeia de valor; indústria química; sustentabilidade ambiental.

\section{Introdução}

O assunto sustentabilidade ambiental tem sido alvo de muitos estudos na literatura científica nacional e internacional. Na área de Gestão da Cadeia de Suprimentos, o conceito de Gestão Verde 
de Cadeia de Valor (GVCV, Green Value Chain Management) (SANTA-EULALIA et al, 2011) tem sido apontado como sendo promissor no sentido de reduzir o impacto ambiental de empresas por meio da introdução de práticas de gestão consciente nas áreas de abastecimento, produção, distribuição, desenvolvimento de produtos, tecnologia de informação, dentre outras.

As pesquisas na área têm apontado que, ao se implementar esse tipo de práticas de gestão, pode-se obter ganhos relacionados ao meio ambiente (e.g. redução de emissões de gás de efeito estufa, de resíduos, etc.) e, ao mesmo tempo, de forma interessante, a adoção dessas práticas pode trazer ganhos também econômicos (redução de custos, aumento de fatias de mercado, etc.) (CHIE e SHIH, 2007; ZHU et al, 2005).

No entanto, os principais estudos empíricos tem sido realizado em grandes empresas, sendo que na área de Pequenas e Médias Empresas (PME) muitas questões ainda permanecem abertas (SANTA-EULALIA et al, 2011; PRUD'HOMME, 2009; TAMRI, 2008; TAMLYN, 2007). Em especial, pouco se sabe sobre as PMEs do setor químico, sendo que este tipo de empresa está comumente associado à problemas de meio ambiente, devido as características inerentes de seus produtos e processos. O setor químico colabora muito com o desenvolvimento da qualidade de vida do ser humano, mas também é um gerador de resíduos e emissões, as quais podem ser prejudiciais ao meio ambiente ou aos consumidores. Isto exige cuidados especiais na gestão da cadeia de suprimentos.

Sabe-se que a química será uma das ciências que mais contribuirá para o desenvolvimento sustentável, por meio de processos que visam à economia dos recursos naturais, buscando soluções para indústrias que enfrentam riscos com a fabricação de substâncias químicas de forma que alcancem tanto a segurança dos trabalhadores, como da sociedade, transformando o risco em benefício (SLEZYNGER e FIGUEIREDO, 2012). No entanto, essas transformações devem vir acompanhadas de um repensar consciente de toda a cadeia de valor, desde a extração da matériaprima ao consumo final e descarte.

Visando contribuir para a redução desta lacuna, Santa-Eulalia et al (2011) iniciou um trabalho para estudar como as PME de Québec, Canadá, estão vivendo a transformação para uma economia ecologicamente mais consciente, do ponto de vista da gestão da cadeia de valor. Tal estudo levanta quais são os principais motivadores para a adoção de uma série de práticas de gestão verde da cadeia de valor e quais são os impactos percebidos pelos gestores das empresas quanto a ganhos de performance ambiental e econômica. Este trabalho despertou o interesse em aplicar a mesma pesquisa no Brasil, um país que possui um índice de crescimento no setor químico e um número significativo de PME, conforme dados do Sebrae (2009).

Neste contexto, a cidade de Uberaba está em desenvolvimento, já possui importantes empresas multinacionais do setor químico que são referência. Além disso, o Governo Municipal 
está investido na criação do quarto Distrito Industrial que tem atraído mais empresas deste setor. Com a expansão e o aumento de indústrias químicas na cidade, levantou-se a preocupação quanto à geração de resíduos e seu descarte.

Sendo assim, o objetivo deste trabalho é analisar empiricamente, por meio de uma série de dez estudos de caso, o processo de adoção de práticas sustentáveis em PME do setor químico em Uberaba. Para tanto, levanta-se os principais motivadores de adoção, do tipo de práticas sendo adotadas e os possíveis impactos econômicos e ambientais percebidos pelos gestores destas empresas. Utiliza-se, para tanto, o modelo conceitual de Santa-Eulalia et al (2011) para que, uma vez o estudo canadense finalizado e publicado, que se possa realizar uma análise comparativa do tipo norte-sul.

O artigo está organizado da seguinte forma: após esta introdução, a Seção 2 apresenta uma breve revisão da literatura e introduz o quadro conceitual empregado nesta pesquisa, sendo que a Seção 3 explica os procedimentos metodológicos utilizados; já a Seção 4 apresenta e discute os resultados do estudo de dez empresas; e a Seção 5 lança as conclusões e trabalhos futuros.

\section{Fundamentação teórica e quadro conceitual}

A sustentabilidade tornou-se foco para muitas empresas. Pode-se dizer que algo é sustentável do ponto de vista ambiental quando consegue reduzir a pressão sobre o meio ambiente de tal forma que ele possa se renovar permitindo seu uso pelas gerações posteriores (GROZNIK e ERJAVEC, 2012). Não basta o produto final ser sustentável, o consumidor entende que o processo de formação do produto também deve ser baseado em desenvolvimento sustentável (SEURING et al, 2008). Isto força as empresas a desenvolverem um modelo de gestão também sustentável, desde uma simples práticas, até grandes atitudes.

A motivação para adoção de práticas sustentáveis tem sido cada vez mais perceptível. O relatório co-escrito pelo CDP, anteriormente Carbon Disclosure Project, e pela empresa de serviços profissionais PwC (2013), fornece uma das principais avaliações mundiais sobre o comportamento corporativo frente à mudança climática, com foco em grandes empresas. Destaca-se como clientes, fornecedores, empregados, governos e a sociedade em geral estão se tornando mais exigentes em relação aos negócios, ou seja são fatores importantes motivadores de mudanças na postura das empresas.

Nikbakhsh (2009) ressalta, se tornar verde pode ser visto como uma importante estratégia de agregação de valor e favorecer a imagem da empresa (o que pode vir a refletir em seu desempenho econômico) bem como trazer benefícios econômicos diretos (como a redução de custos de matérias primas, redução de riscos de multas por geração de resíduos, melhora da produtividade, dentre outras). 
Tais ganhos são tipicamente evidenciados em grandes empresas, especialmente as multinacionais. No contexto das PME, segundo Santa-Eulalia et al (2011), existe a dificuldade de se apoiar práticas sustentáveis. As PMEs são normalmente motivadas por medidas impostas por órgãos governamentais e regulamentos, ou ainda por motivações pessoais de seus líderes, os quais possuem posturas inovadoras e visionárias. No entanto, pouco são os estudos sobre práticas de gestão verde em cadeias de valor neste tipo de empresas.

O estudo pioneiro apresentado em Santa-Eulalia et al (2011) aborda três conjuntos de elementos que tem relação com o uso de práticas de gestão verdes de cadeia de valor: motivações para a adoção de novas práticas; quais são tais práticas ao longo da cadeia de valor (incluindo abastecimento, produção, distribuição, desenvolvimento de produtos, tecnologia de informação e adoção de indicadores de performance adequados) e, uma vez adotadas, quais seriam os impactos percebidos na performance ambiental e econômica. Como este modelo será empregado no presente estudo, apresenta-se, a seguir, um resumo do mesmo.

Neste modelo, tudo começa no processo de adoção das práticas. São muitos os estudos a fim de entender como se dá a difusão de novos produtos e/ou práticas no mercado, impulsionados pelo interesse da maioria das empresas, assim como também do governo) para disseminação e estruturação de regulamentações, por exemplo). A teoria da difusão (ROGERS, 2003), apesar de complexa, é normalmente usada para explicar como uma nova ideia é aceita pelo mercado. No caso das práticas verdes, sabe-se que o processo de adoção tem motivações externas, como pressões provenientes de clientes, fornecedores e ONGs (CHIE e SHIH, 2007) e dos governos (LEE, 2008; CHIE e SHIH, 2007). Isso leva a primeira hipótese de pesquisa H1: A motivação externa está positivamente relacionada coma a adoção de práticas $G V C V$.

Rogers observou como as mudanças são difundidas através da sociedade ao longo do tempo, desvendando algumas barreiras assim como facilitadores para o processo de difusão de inovações. São cinco as características definidas por Rogers (1983) que influenciam na tomada de decisão em aprovar ou rejeitar uma inovação, são elas: i) vantagem relativa: como uma inovação é considerada superior às anteriores em termos de custos, prestígio social, e satisfação, por exemplo; ii) compatibilidade: o grau no qual uma inovação se enquadra (i.e., é compatível) na vida daquele que a adota; iii) complexidade/simplicidade: se complexo for entender ou usar uma inovação, pouco provável será sua aceitação; iv) experimentabilidade: a facilidade que se tem de testar, i.e. entrar em contato com uma inovação antes de sua adoção; v) observabilidade: a percepção dos resultados da adoção da inovação por terceiros.

Desta forma, mesmo na ausência de motivações externas (e.g. pressões governamentais) como mencionadas na primeira hipótese, a motivação interna pode existir e influenciar a adoção de 
práticas sustentáveis como forma de inovação nas PMEs. Isso levaria à segunda hipótese H2: $A$ motivação interna está positivamente relacionada com a adoção de práticas GVCV.

Outro fator importante para a pesquisa é o desempenho organizacional (CHIE e SHIH, 2007; ZHU et al, 2005;. HERVANI et al, 2005). Melhores performances foram constatadas por Chie e Shih (2007) em empresas que adotaram práticas sustentáveis. A performance econômica está relacionada aos custos (e.g. consumo de energia, água, e custos envolvidos na prevenção de alguma multa resultante de acidentes ambientas); aos lucros (e.g. decorrentes da conquista de novos produtos e nichos de mercado); e aumento do market share (e.g. pelo apelo dos consumidores a produtos verdes). A performance ambiental está relacionada com o impacto que as atividades de uma empresa causam ao meio ambiente, entre eles os principais são: as emissões de poluentes (principalmente gases do efeito estufa), consumo de energia, o consumo de recursos naturais, eliminação de resíduos e preservação da biodiversidade. Isso leva a duas últimas hipóteses H3: Práticas GVCV estão positivamente relacionadas com a performance ambiental; e Hipótese H4: Práticas GVCV estão positivamente relacionadas com a performance econômica.

Alcançar um equilíbrio entre as performances ambiental e econômica é uma séria preocupação para as empresas que buscam implementar as práticas sustentáveis (CHIE e SHIH, 2007; ZHU et al, 2005). Assim, é importante avaliar tanto as performances econômicas quanto as ambientais, considerando se ainda o aspecto de que as PME's possuem recursos financeiros limitados (RAO, 2002). Por sua vez, se a hipótese H4 for confirmada será um incentivo às empresas mais resistentes a adoção de práticas verdes, dado que é possível potencializar receitas e lucros através de práticas GVCV, como evidenciado também pelo relatório CDP \& PwC (2013).

Todas as quarto hipóteses estão representadas no modelo conceitual apresentado na Figura 1, segundo Santa-Eulalia et al (2011). 
Figura 1 - Quadro Conceitual adotado

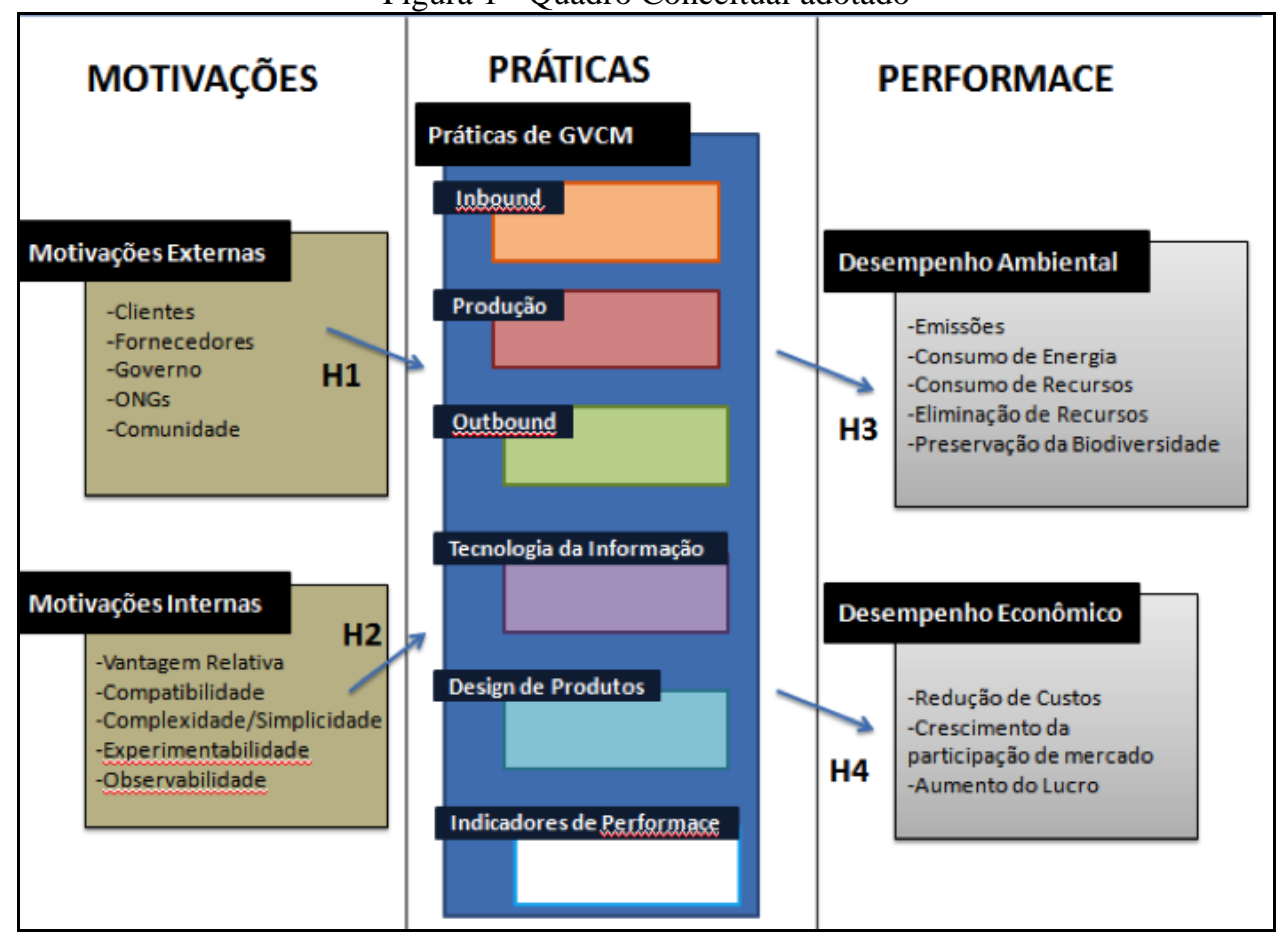

Fonte: Santa-Eulalia et al (2011)

As práticas verdes selecionadas neste estudo (parte central da Figura 1) são baseadas em uma revisão da literatura na área, incluindo pesquisas qualitativas e empíricas, as quais foram apresentada em Santa-Eulalia et al (2011).

Rao et al (2005) utilizaram a adoção de práticas verdes na logística de entrada (inbound), no processo de produção e na logística de saída (outbound) para medir o impacto na competitividade e desempenho econômico das empresas no sudeste asiático. Bruke e Gaughran (2006) evidenciam que, apesar de a Tecnologia da informação (TI) ser um aliado importante para a GVCV na gestão dos impactos ambientais pela coleta armazenagem e processamentos de dados que geram informações importantes para favorecer melhorias, seu uso é muito restrito entre as PME. Essa limitação é atribuída a pouca oferta de sistemas voltados as PME e os que existem no mercado são normalmente caros e limitados em recursos, assim como na sua assistência. Apesar da pouca bibliografia sobre o impacto do uso de TI em iniciativas de GVCM para PME, foi decidido testar o conceito no modelo.

Práticas relacionadas ao design de produtos verdes tem sido bastante reconhecidas pelo mercado como uma prática comercial interessante, sendo que sua influência sobre a performances ambiental pode ser vista em Hong et al (2009).

No entanto, Rao et al (2009) demonstram que as PME podem melhorar seu desempenho ambiental e econômico por meio do uso adequado de indicadores de performance pertinentes, mesmo para as empresas que não possuem nenhum sistema de gestão ambiental implementado. Isto 
permitiria as empresas medirem sua performance e identificar a distância entre seu real desempenho e os padrões industriais base, normas e de concorrentes.

A aplicação do modelo conceitual de PME de Santa-Eulalia et al (2011) faz-se pertinente no cenário brasileiro, pois as PME no Brasil registram uma participação marcante em sua economia, pois elas representam cerca de $99 \%$ do universo das empresas nacionais e, juntas, respondem por cerca de $60 \%$ da mão de obra e por $20 \%$ do PIB brasileiro (MATOS et al, 2005).

Neste trabalho, o setor químico é estratégico na economia nacional, tem presença marcante nas cadeias produtivas locais, sendo que o Brasil ocupa a oitava posição mundial na fabricação de produtos químicos (WONGTSCHOWSKI, 2011). Para a ABIQUIM (Associação Brasileira de Indústria Química) a indústria química é um dos mais importantes setores da economia brasileira (ABIQUIM, 2013).

De acordo com BRASIL (2006), a indústria química pode ser classificada em dois grupos: de uso industrial e de uso final. Nesta pesquisa, as empresas entrevistadas foram do grupo de produtos químicos de uso final: cosméticos, higiene pessoal, tintas, vernizes, esmaltes, sabão, limpeza. Este grupo de produtos de uso final é importante para a pesquisa por ser uma classe de muito consumo e gerar resíduos, sendo produtos de fabricação renovável e não renovável. A indústria química fabrica uma série de produtos para diversos setores, os que mais se destacam são: fabricação de tintas, produtos de limpeza e cosméticos (WONGTSCHOWSKI, 2011).

Sendo assim, com base no quadro conceitual da Figura 1, apresenta-se a seguir como o presente estudo organiza o ferramental metodológico visando estudar o processo de adoção de práticas de GVCV em PME do setor químico.

\section{Metodologia de Pesquisa}

O quadro conceitual utilizado neste presente trabalho e suas hipóteses são a base para as seguintes questões de pesquisa:

- Quais são as motivações das PME do setor químico de Uberaba a adoção de práticas de GVCV?;

- Quais são as práticas adotadas por tais empresas?;

- Qual o impacto percebido na performance ambiental e econômica da adoção de tais práticas?;

- As hipóteses H1, H2, H3 e H4 fazem sentido, do ponto de vista de um estudo qualitativo preliminar?.

Com o objetivo de abordar tais indagações, o presente trabalho é de natureza "exploratória" e faz uso da pesquisa dita "estudo de caso" (YIN, 2009; SILVA e MENEZES, 2005) múltiplo, com 
uma amostra de dez empresas de Uberaba. Todas as entrevistas usaram o mesmo questionário semiaberto de Santa-Eulalia et al (2011). As entrevistas foram realizadas durante o ano de 2013.

Uberaba está centrada na região do Triângulo Mineiro, no estado de Minas Gerais, e possui localização estratégica, sendo que a mesma se encontra a menos de 500 quilômetros de distância de importantes pólos administrativos, financeiros e consumidores. Conta com conexão, ferroviária e um grande número de empresas da indústria química, de fertilizantes, distribuição de combustíveis e transportadoras (UBERABA, ([201-]).

Em meio a uma economia diversificada, Uberaba possui um bom desenvolvimento econômico é o $7^{\circ}$ maior gerador de empregos formais do estado (2010); PIB per capita anual: R\$ 21.279,05 (2008); maior pólo de fertilizantes fosfatados da América Latina. Possui três Distritos Industriais em funcionamento e três mini-distritos para Pequenas Empresas (UBERABA, ([201-]).

A cidade se destaca em diversos setores: alimentício, moveleiro, calçadista, químico e de fertilizantes, considerado um dos 100 melhores centros industriais do Brasil. Com investimento em desenvolvimento, Uberaba é a $7^{\mathrm{a}}$ maior economia de Minas Gerais, de acordo com a Fundação João Pinheiro (2010) em parceria com o IBGE.

A seleção das empresas partiu da base de requisitos utilizada no estudo canadense de SantaEulalia et al (2011) para PME, as quais deve ter faturamento anual inferior a 50.000.000,00 CAD\$ (aproximadamente R $\$ 107.000 .000,00)$ e até 500 empregados. Como o Sebrae define PME como sendo até 500 funcionários, ambas as definições estariam coerentes. Além disso, conforme a Lei 9.841/99 ("Estatuto da Microempresa e da Empresa de Pequeno Porte"), que estabelece ter receita bruta anual inferior a $\mathrm{R} \$ 1.2000 .000,00$.

A seleção das indústrias, do setor de química de Uberaba se deu com base na resolução da Classificação Nacional de Atividades Econômicas - CNAE 2.0 (BRASIL, 2006). Visando se aproximar das empresas estudadas por Santa-Eulalia et al (2011), foram selecionados dois grupos: fabricação de sabões, detergentes, produtos de limpeza, cosméticos e higiene pessoal; e o grupo de fabricação de tintas, vernizes e produtos afins.

Para se chegar às indústrias químicas de Uberaba, foi feito um rastreamento por meio do site da ABIQUIM e no Sindicato dos Trabalhadores nas Indústrias Químicas e Farmacêuticas de Uberaba e Região (STIQUIFAR). Neste, estão cadastradas 16 indústrias que se classificam nestes dois grupos. Com o contato das indústrias repassado pelo sindicato, estas 16 foram convidadas a participar da pesquisa por meio de ligação telefônica e e-mail. Conseguiu-se entrevista com 10 empresas, totalizando mais de $60 \%$ da população de empresas do setor estudado na região.

Antes de iniciar as entrevistas, o questionário a ser empregado foi submetido ao Comitê de Ética da Universidade Federal do Triângulo Mineiro (UFTM) para aprovação e garantia de sigilo da pesquisa. As entrevistas foram realizadas na sede das indústrias com os gestores responsáveis, 
conforme autorização dos participantes e todas foram gravadas em áudio. Posteriormente, foram transcritas nas mesmas palavras do entrevistado. Os entrevistados eram, preferencialmente, o líder da empresa, normalmente o diretor geral, ou presidente, pois os mesmos possuem uma visão ampla do funcionamento da empresa. Ou ainda, o diretor geral ou presidente da empresa poderia indicar funcionários com visão geral das práticas de gestão verde já implementadas ou sendo implementadas.

\section{Resultados}

Esta seção discute os principais resultados da pesquisa. Primeiramente, apresenta-se cada empresa individualmente para, em seguida, discutir a amostra toda na tentativa de resumir e quantificar os dados de natureza qualitativa extraídos de tais casos.

\subsection{Estudos de Casos}

O perfil dos entrevistados é de gestor ou responsável indicado pelo proprietário.

A Empresa 1 possui menos de 25 funcionários e seus mercados atendidos são atacado para lojistas de Uberaba e Região. Um breve resumo dos principais elementos investigados na empresa 1 estão disponíveis na Tabela 1.

Tabela 1 - Principais Elementos das Entrevistas para a Empresa 1.

\begin{tabular}{cl}
\hline Tópicos & Resumo dos Principais Elementos Abordados na Pesquisa \\
\hline Geral & A empresa não tem como objetivo estabelecer metas para as iniciativas \\
& sustentáveis, apenas cumpre com a legislação exigida \\
Motivações de adoção & Caso existam produtos fornecidos que sejam sustentáveis e com preço acessível a \\
& indústria optaria nesta versão \\
Práticas sustentáveis & Não adota \\
Desempenho & Não tem como medir o desempenho \\
Outras & Acredita que a iniciativa deveria vir do fornecedor \\
\hline
\end{tabular}

Fonte: Pesquisa de campo (2014)

O entrevistado justificou que, devido o alto volume de trabalho presente, as iniciativas sustentáveis implementadas visam apenas o cumprimento da legislação em vigor.

A Empresa 2 possui menos de 25 funcionários e seus mercados atendidos são regional e nacional Os principais elementos investigados na empresa estão resumidos na Tabela 2.

Tabela 2 - Principais Elementos das Entrevistas para a Empresa 2

\begin{tabular}{|c|c|}
\hline Tópicos & Resumo dos Principais Elementos Abordados na Pesquisa \\
\hline Geral & $\begin{array}{l}\text { Existe a preocupação com a sustentabilidade, a indústria tem consciência de que } \\
\text { algumas atitudes são feitas, mas o mínimo que faz é devido exigência legal }\end{array}$ \\
\hline Motivações de adoção & $\begin{array}{l}\text { A motivação externa vem do fornecedor que oferece um produto acessível e menos } \\
\text { agressor que um produto comum. Motivação interna é cultural, as práticas são feitas } \\
\text { conforme conceitos adquiridos como economia de água, energia e ainda dá abertura } \\
\text { aos funcionários para opinarem sobre melhorias no ambiente de trabalho. }\end{array}$ \\
\hline Práticas sustentáveis & Seleciona matéria-prima que respeita o meio ambiente \\
\hline Desempenho & Não mede o desempenho mas acredita que seja positivo na relação financeira e que \\
\hline
\end{tabular}


ajuda na preservação da biodiversidade

Outras

A principal barreira é a falta de incentivos públicos

Fonte: Pesquisa de campo (2014)

O entrevistado ressaltou que a empresa é pequena e que faltam recursos para inovar nas práticas de sustentabilidade.

A Empresa 3 possui de 25 a 50 funcionários e seus mercados atendidos são regional, Triângulo Mineiro e parte de Goiás. Na Tabela 3, apresenta-se um principais elementos investigados na empresa 3 .

Tabela 3 - Principais Elementos das Entrevistas na Empresa 3.

\begin{tabular}{cl}
\hline Tópicos & \multicolumn{1}{c}{ Resumo dos Principais Elementos Abordados na Pesquisa } \\
\hline Geral & $\begin{array}{c}\text { Não utiliza de produtos tóxicos que possam agredir o meio ambiente, os resíduos } \\
\text { tem aterro próprio liberado pela secretaria de meio ambiente }\end{array}$ \\
Motivações de adoção & A motivações são devidas as facilidades de adoção e simplicidade em fabricar sem \\
& agredir o meio ambiente \\
Práticas sustentáveis & Economia interna de uso de papel, água, energia \\
Desempenho & Não mede o desempenho econômico ou ambiental, mas acredita que há aumento na \\
& rentabilidade e preservação do meio ambiente \\
Outras & A principal barreira é a falta de incentivos públicos destinados para as PMEs
\end{tabular}

Fonte: Pesquisa de campo (2014)

A indústria fabrica produtos que não agridem o meio ambiente, já é um produto diferenciado do comumente encontrado no mercado e com preço acessível. A dificuldade em fazê-lo está no alto valor que pagam para o descarte dos resíduos. A empresa esperaria que parte desta tarefa fosse do governo, assim reduziria-se os gastos para facilitar investimentos em outras práticas de gestão verde.

A Empresa 4 possui mais de 50 funcionários e seu mercado atendido é regional. A seguir, na tabela 4 apresenta-se um curto resumo dos principais elementos investigados na empresa 4.

Tabela 4 - Principais Elementos das Entrevistas na Empresa 4.

\begin{tabular}{cl}
\hline Tópicos & \multicolumn{1}{c}{ Resumo dos Principais Elementos Abordados na Pesquisa } \\
\hline Geral & Possui as licenças para funcionamento e ainda buscam parcerias com programas de \\
& sustentabilidade \\
Motivações de adoção & Motivação cultural \\
Práticas sustentáveis & Escolhe fornecedores que tenham responsabilidade social e sustentabilidade com \\
& produtos que respeitem o meio ambiente \\
Desempenho & Não mede o desempenho mas acredita que seja positivo na relação financeira com a \\
& redução de custos e que ajuda na preservação da biodiversidade \\
Outras & A principal barreira é a falta de incentivos públicos \\
\hline
\end{tabular}

Fonte: Pesquisa de campo (2014)

O proprietário tem interesse pessoal por iniciativas sustentáveis e busca inovar para conseguir melhor aproveitamento de água, energia, descarte dos resíduos. Não aguarda iniciativas do governo, mas tenta mudar algo para que seja possível a criação de novos incentivos.

A Empresa 5 possui menos de 25 funcionários e seu mercado atendido é regional. $\mathrm{Na}$ Tabela 5, apresenta-se os principais elementos investigados na empresa 5. 
Tabela 5 - Principais Elementos das Entrevistas na Empresa 5.

\begin{tabular}{cl}
\hline Tópicos & \multicolumn{1}{c}{ Resumo dos Principais Elementos Abordados na Pesquisa } \\
\hline Geral & Atende a legislação exigida para funcionamento \\
Motivações de adoção & Cultural e visibilidade de mudança \\
Práticas sustentáveis & Algumas práticas são feitas na linha de produção, evitando o desperdício e descarte \\
& de produto na natureza e na distribuição com a logística reversa onde os clientes, a \\
& partir da segunda compra, pagam apenas pelo produto ganhando desconto na \\
embalagem que retornou. & Acredita que pode aumentar a rentabilidade e preservar a biodiversidade \\
Desempenho & A principal barreira é a falta de incentivos públicos \\
Outras &
\end{tabular}

Fonte: Pesquisa de campo (2014)

Devido o alto volume de trabalho e a falta de funcionários, as iniciativas sustentáveis ficaram, até o momento, apenas no cumprimento da legislação.

A Empresa 6 possui menos de 25 funcionários e seu mercado atendido é regional. $\mathrm{Na}$

Tabela 6, apresenta-se os principais elementos investigados na empresa 6.

Tabela 6 - Principais Elementos das Entrevistas na Empresa 6.

\begin{tabular}{cl}
\hline Tópicos & \multicolumn{1}{c}{ Resumo dos Principais Elementos Abordados na Pesquisa } \\
\hline Geral & Não possui políticas de sustentabilidade, mas se preocupa com pequenas atitudes \\
Motivações de adoção & Motivação interna está relacionada com a vantagem que o produto oferece para o \\
& mercado e traz retorno financeiro positivo para a indústria. \\
Práticas sustentáveis & Utiliza de algumas práticas para contribuir com a sustentabilidade que são a compra \\
& de matéria-prima e uso da internet como redução do uso de papel \\
Desempenho & Acredita que pode reduzir custos e, em relação ao meio ambiente, pode reduzir a \\
& geração de resíduos e preservar a biodiversidade \\
Outras & A principal barreira é a falta de incentivos públicos \\
\hline
\end{tabular}

Fonte: Pesquisa de campo (2014)

Existe a preocupação e vontade de se aplicar atitudes sustentáveis, mas acredita-se que não deve ser apenas por parte da indústria, uma vez que se faz a separação dos resíduos mas a coleta deste lixo o leva para o mesmo local e não há separação.

A Empresa 7 possui menos de 25 funcionários e seu mercado atendido é regional. Na Tabela 7, apresenta-se os principais elementos investigados na empresa 7.

Tabela 7 - Principais Elementos das Entrevistas na Empresa 7.

\begin{tabular}{cl}
\hline Tópicos & \multicolumn{1}{c}{ Resumo dos Principais Elementos Abordados na Pesquisa } \\
\hline Geral & Atende a legislação exigida para funcionamento \\
Motivações de adoção & Motivação é cultural devido as práticas em casa, no dia-a-dia leva também para a \\
& empresa atitudes como economia e energia e água \\
Práticas sustentáveis & Praticas são feitas no abastecimento com a seleção de matérias-primas; na produção \\
& evitando o acúmulo de resíduos e uso de tecnologia da informação no lugar de \\
& papel \\
Desempenho & Acredita que pode haver redução de custos para o desempenho econômico e \\
& preservação da biodiversidade \\
Outras & A principal barreira é a falta de incentivos públicos \\
\hline
\end{tabular}

Fonte: Pesquisa de campo (2014)

A indústria é pequena e faltam recursos para inovar nas práticas de sustentabilidade. 
A Empresa 8 possui de 25 a 50 funcionários e seu mercado atendido é regional e alguns estados. $\mathrm{Na}$

Tabela 6, apresenta-se os principais elementos investigados na empresa 8.

Tabela 8 - Principais Elementos das Entrevistas na Empresa 8

\begin{tabular}{ll}
\hline \multicolumn{1}{c}{ Tópicos } & \multicolumn{1}{c}{ Resumo dos Principais Elementos Abordados na Pesquisa } \\
\hline Geral & Atende a legislação exigida para funcionamento \\
Motivações de adoção & A motivação é interna, está relacionada com a vantagem que o produto oferece em \\
& relação ao produto comum. \\
Práticas sustentáveis & Acredita-se que as práticas são mais eficientes se aplicadas na linha de produção \\
Desempenho & Acredita que pode aumentar a rentabilidade, reduzir resíduos e preservar a \\
& biodiversidade \\
Outras & A principal barreira é a falta de incentivos públicos e falta de fornecedores
\end{tabular}

Fonte: Pesquisa de campo (2014)

Devido o alto volume de trabalho as iniciativas sustentáveis ficam apenas no cumprimento da legislação.

A Empresa 9 possui menos de 25 funcionários e seu mercado atendido é regional. Na Tabela 9, apresenta-se os principais elementos investigados na empresa 9.

Tabela 9 - Principais Elementos das Entrevistas na Empresa 9.

\begin{tabular}{cl}
\hline Tópicos & \multicolumn{1}{c}{ Resumo dos Principais Elementos Abordados na Pesquisa } \\
\hline Geral & Atende a legislação exigida para funcionamento \\
Motivações de adoção & Motivação cultural, ainda que faltem incentivos públicos, pode-se fazer alguma \\
& coisa internamente \\
Práticas sustentáveis & As práticas são na linha de produção com a escolha de matéria-prima, na produção \\
& com redução de resíduos e na distribuição com uso da tecnologia \\
Desempenho & Acredita que pode aumentar a rentabilidade e preservar a biodiversidade \\
Outras & A principal barreira é a falta de fornecedores \\
\hline
\end{tabular}
Fonte: Pesquisa de campo (2014)

Novamente, devido o alto volume de trabalho as iniciativas sustentáveis ficam apenas no cumprimento da legislação, mas buscam formas de melhoria para o desempenho ambiental e que tragam resultados como redução de custos.

A Empresa 10 possui menos de 25 funcionários e seu mercado atendido é regional, prestadores de serviço e varejo. Na Tabela 10, apresenta-se os principais elementos investigados na empresa 5.

Tabela 10 - Principais Elementos das Entrevistas na Empresa 10.

\begin{tabular}{cl}
\hline Tópicos & \multicolumn{1}{c}{ Resumo dos Principais Elementos Abordados na Pesquisa } \\
\hline Geral & Atende a legislação exigida para funcionamento \\
Motivações de adoção & Motivação externa, deveria haver mais incentivos públicos \\
Práticas sustentáveis & As práticas são na linha de produção com a escolha de matéria-prima, redução de \\
& resíduos e na distribuição com uso da tecnologia \\
Desempenho & Acredita que pode aumentar a rentabilidade e preservar a biodiversidade \\
Outras & A principal barreira é a falta de fornecedores que preços acessíveis \\
\hline
\end{tabular}

Fonte: Pesquisa de campo (2014) 
Acredita-se que o investimento em práticas sustentáveis ainda são muito altos, substituir a embalagem de produto comum por outra que seja biodegradável ainda é caro e necessita de investimentos em certificações.

\subsection{Panorama da Amostra}

Nesta subseção, apresenta-se uma compilação dos dez estudos de caso, visando quantificar os principais dados obtidos. Primeiramente, um resumo das empresas estudadas é apresentado na Tabela 11.

Tabela 11 - Principais Características das Empresas Entrevistadas

\begin{tabular}{|c|c|c|c|}
\hline Empresa & $\begin{array}{l}\text { Àrea de atuação (CNAE) } \\
\text { Seção C, divisão } 20\end{array}$ & $\begin{array}{l}\text { Quantidade de } \\
\text { funcionários }\end{array}$ & Principais produtos \\
\hline Empresa 1 & Grupo 207 & menos de 25 & Tintas e vernizes \\
\hline Empresa 2 & 206 & menos de 25 & $\begin{array}{c}\text { Produtos de beleza e higiene } \\
\text { pessoal }\end{array}$ \\
\hline Empresa 3 & 207 & de 25 a 50 & $\begin{array}{l}\text { Látex e revestimentos } \\
\text { acrílicos }\end{array}$ \\
\hline Empresa 4 & 206 & mais de 50 & $\begin{array}{l}\text { Produção de cosméticos e } \\
\text { artigos de higiene pessoal }\end{array}$ \\
\hline Empresa 5 & 206 & menos de 25 & Produtos químicos de limpeza \\
\hline Empresa 6 & 207 & menos de 25 & Tintas \\
\hline Empresa 7 & 206 & menos de 25 & Higiene pessoal \\
\hline Empresa 8 & 206 & de 25 a 50 & Higiene pessoal \\
\hline Empresa 9 & 206 & menos de 25 & Higiene pessoal \\
\hline Empresa 10 & 206 & menos de 25 & Cosméticos para profissionais \\
\hline
\end{tabular}

Nota 1: Grupo 207, segundo CNAE: Fabricação de Tintas, vernizes, esmaltes, lacas e produtos afins.

Nota 2: Grupo 206, segundo CNAE: Fabricação de sabões, detergentes, produtos de limpeza, cosméticos, produtos de perfumaria e higiene pessoal

Fonte: Pesquisa de campo (2014)

A Tabela 3, a seguir, resume algumas características das indústrias que fizeram parte da amostra deste estudo.

Tabela 12 - Características das empresas estudadas.

\begin{tabular}{l|cc}
\multicolumn{1}{c}{ Questões } & \multicolumn{2}{c}{ Total } \\
\hline A empresa possui uma política, planos ou procedimentos internos e & $90 \%$ & $10 \%$ \\
explícitos de gestão verde? & Não & Sim \\
A empresa fornece recursos e desenvolve competências necessárias para & $60 \%$ & $40 \%$ \\
atingir os objetivos ambientais? & Não & Sim \\
A empresa é certificada "verde" ou está a caminho de obter uma certificação & $0 \%$ & $100 \%$ \\
(por exemplo, ISO 14000, EMAS)? & Não & $80 \%$ \\
Existe um gestor/ coordenador para gerenciar as práticas "verdes"? & $20 \%$ & Simo \\
A empresa tem como mercado-alvo, entre outros, os consumidores de & $80 \%$ & $20 \%$ \\
produtos "verdes"? & Não & Sim \\
Há quanto tempo sua empresa investe recursos em gestão verde? & $60 \%$ & $40 \%$ \\
\end{tabular}

Fonte: Pesquisa de campo (2013)

Este grupo pesquisado corresponde a $62 \%$ das indústrias químicas da cidade de Uberaba, na área de higiene pessoal, limpeza e tintas. Os principais mercados que atendem são a região (Triângulo Mineiro) e algumas abrangem outros estados. 
Segundo a pesquisa, nenhuma das indústrias possui política explícita de gestão verde, mas duas delas possuem procedimentos internos que vão além do exigido pelo governo. $60 \%$ das indústrias entrevistadas entendem que, mesmo que seja um requisito para funcionamento, o fato de tratar os resíduos ou comprar matérias-primas que não agridam o meio ambiente já são recursos destinados a atingir os objetivos ambientais.

As respostas indicam que todas as indústrias possuem as licenças ambientais e os documentos exigidos para o funcionamento. A maioria das indústrias possui um coordenador para cuidar das práticas de sustentabilidade, este coordenador em alguns casos é o próprio gestor, em outros casos é indicado um responsável, uma vez que são fiscalizados pelos órgãos competentes.

Mesmo compreendendo que é uma tendência de mercado os consumidores aderirem aos produtos ecologicamente corretos, as indústrias pesquisadas, ainda em sua maioria, não visam esse público. O tempo de investimento das indústrias em sustentabilidade está associado ao tempo de funcionamento, uma vez que para iniciar as atividades é obrigatório que se cumpram algumas exigências ambientais.

A Tabela 13 apresenta as principais motivações dos empresários da cidade a praticarem a sustentabilidade, segundo o modelo conceitual empregado nos estudos de caso.

Tabela 13 - Motivações de adoção das práticas sustentáveis.

\begin{tabular}{|c|c|c|c|c|c|c|c|c|c|c|c|}
\hline \multirow{2}{*}{ Questões } & \multicolumn{10}{|c|}{ Respostas das Indústrias } & \multirow{2}{*}{ Total } \\
\hline & 1 & 2 & 3 & 4 & 5 & 6 & 7 & 8 & 9 & 10 & \\
\hline \multicolumn{12}{|c|}{$\begin{array}{l}\text { Em sua opinião, quais foram as motivações da empresa na } \\
\text { adoção de políticas sustentáveis? }\end{array}$} \\
\hline Vantagem relativa ao modelo anterior & & & & & & & & & & & $0 \%$ \\
\hline Facilidade / simplicidade na adoção & & & $\mathrm{X}$ & & & & & & $\mathrm{X}$ & & $20 \%$ \\
\hline Experimentação & & & & & & & & & & & $0 \%$ \\
\hline Visibilidade das vantagens da mudança & & & & & $\mathrm{X}$ & $X$ & & & & & $20 \%$ \\
\hline Clientes / consumidores & & & & & & & & & & & $0 \%$ \\
\hline Fornecedores & & $\mathrm{X}$ & & & & & & & & & $10 \%$ \\
\hline Governo & & & & & & & & & & $\mathrm{X}$ & $10 \%$ \\
\hline ONGs & & & & & & & & & & & $0 \%$ \\
\hline Comunidade & & & & & & & & & & & $0 \%$ \\
\hline Cultura (motivação pessoal) & & & & $X$ & $X$ & $X$ & $X$ & $\mathrm{X}$ & & & $50 \%$ \\
\hline Não adota & $\mathrm{X}$ & & & & & & & & & & $10 \%$ \\
\hline
\end{tabular}

Fonte: Pesquisa de campo (2014)

Tomando como referência os cinco atributos de Rogers (2003), sobre o emprego de algo inovador, como a vantagem relativa, compatibilidade, complexidade/simplicidade, experimentabilidade, observabilidade, a pesquisa aponta qual pode ser rapidamente mais utilizada pelas indústrias. O que mais motiva as práticas é a cultura pessoal, cerca de $50 \%$ dos entrevistados aplicam as práticas sustentáveis, devido à cultura adquirida ao longo da vida. Em outros casos $20 \%$ 
aponta à visibilidade de mudança e a facilidade de adoção do produto como uma motivação para essas práticas. $10 \%$ das empresas acredita que uma boa motivação poderia vir por parte de incentivos do Governo com políticas públicas facilitadoras, do outro lado este incentivo viria dos fornecedores com o fornecimento de matéria-prima sustentável e com preço acessível.

As Tabela 14.1 e Tabela 14.2 colocam em evidência a natureza das práticas adotadas, segundo o modelo conceitual adotado para esta pesquisa.

Tabela 14.1 - Práticas de redução dos impactos ambientais

Sua empresa visa à redução de impactos ambientais em seus processos (abastecimento, produção, distribuição, tecnologia da informação e desenvolvimento de produtos)?

\begin{tabular}{|c|c|c|c|c|c|c|c|c|c|c|c|c|}
\hline Opções & \multicolumn{9}{c|}{ Respostas das Indústrias } & \multirow{2}{*}{ Total } \\
\cline { 1 - 7 }
\end{tabular}

\section{Abastecimento:}

Por exemplo: seleção de matérias-primas e fornecedores que respeitem o meio ambiente; tecnologia verde na fabricação; uso de embalagens padrões e reutilizáveis com fornecedores; identificação e redução de perdas ou pontos de geração de poluição ou emissões; redução de resíduos; logística reversa.

\section{Produção:}

Por exemplo: utiliza tecnologia verde / limpa para transporte, manutenção, iluminação, água reciclada, recuperação de resíduos; reduz as perdas ou pontos de geração de poluição ou emissões; uso de embalagens reutilizáveis; redução de resíduos; logística reversa; ecologia industrial (ex.: reprodução da simbiose de um ecossistema natural); dejetos de uma empresa serve para outra; parque industrial.

Fonte: Pesquisa de campo (2014)

Tabela 14.2 - Práticas de redução dos impactos ambientais.

Sua empresa visa à redução de impactos ambientais em seus processos (abastecimento, produção, distribuição, tecnologia da informação e desenvolvimento de produtos)?

\begin{tabular}{l|l|l|l|l|l|l|l|l|l|l|l|}
\multirow{2}{*}{ Opções } & \multicolumn{7}{|c|}{ Respostas das Indústrias } & \multirow{2}{*}{ Total } \\
\cline { 2 - 9 } & 1 & 2 & 3 & 4 & 5 & 6 & 7 & 8 & 9 & 10 & \\
\hline
\end{tabular}

\section{Distribuição:}

Por exemplo: identifica e reduz as perdas ou pontos de geração de poluição ou emissões; uso de embalagens padrões e reutilizáveis com clientes; utiliza tecnologia verde / limpa arrefecimento dos depósitos, redução de resíduos; logística reversa.

\section{Tecnologia da Informação:}

Por exemplo: trabalho a distância e fornecimento de tecnologias para realizá-lo; servidores e outros equipamentos/computadores; incentivo ao uso de meios eletrônicos ao invés do papel.

$$
\begin{array}{lllllllll}
X & X & X & X & X & X & X & X & 80 \%
\end{array}
$$

\section{Desenvolvimento de Produto:}

Por exemplo: práticas de gestão de eco-design; gestão de projeto do produto de fácil reciclagem ou capacidade de desmontagem.

\section{Geral:}

Por exemplo: sistema produto-serviço - desmaterialização das ofertas de valor da empresas; ex.: compras, financiamento ou 
Em geral, pode-se dizer que as empresas estudadas possuem procedimentos que visam à redução de impactos ambientais, sendo que $60 \%$ aplicam as práticas no abastecimento da indústria e $80 \%$ aplica na produção, mesmo adotando práticas que, na maioria dos casos, são devido às exigências legais. Estas práticas são, por exemplo, recursos para redução de resíduos, gerar menos poluição, reciclar a água que possa ser reutilizada, dentre outras. Além disso, $40 \%$ das empresas utilizam práticas sustentáveis na distribuição de seus produtos, $60 \%$ aplica algum tipo de tecnologia da informação que possa ajudar a gestão ambiental, e 10\% das indústrias não tem perspectiva de redução dos impactos ambientais, sendo que não utilizam nenhuma prática até o momento.

A Figura 2 resume em quais processos mais se aplicam as práticas de sustentabilidade investigadas.

Figura 2 - Setores visados pelas práticas de sustentabilidade empregadas nas empresas estudadas.

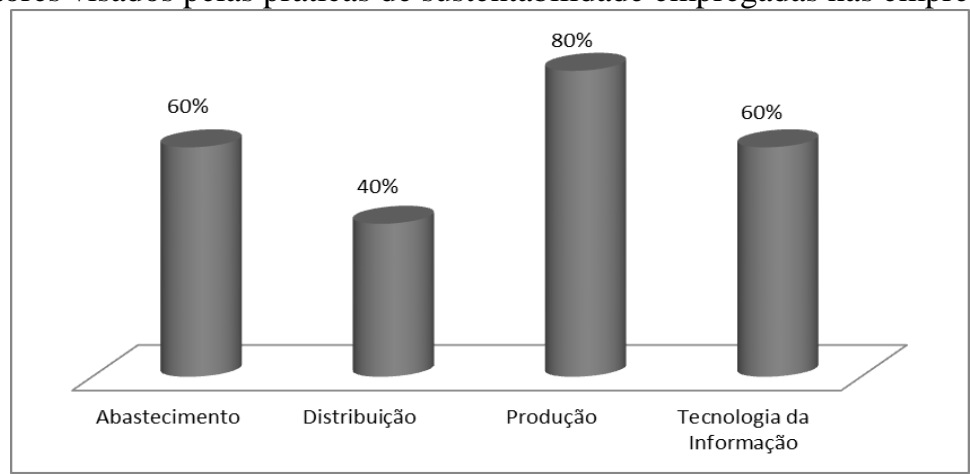

Fonte: Pesquisa de campo (2014)

Com este resultado, é possível observar que o setor de produção é o local em que as pequenas indústrias mais aplicam sustentabilidade. É neste local que se tem maior participação e, com isso, os gestores podem inovar nestas práticas. Este resultado é importante também pois mostra os locais de maior demanda para possíveis inovações e fornecimento de tecnologias para suprir a necessidade de prática da sustentabilidade.

A Tabela 15 mostra as práticas, elencadas pelo entrevistado, adotadas pelas indústrias entrevistadas conforme dados disponíveis no roteiro.

Tabela 15 - Detalhamento das práticas adotadas elencadas pelos entrevistados.

\begin{tabular}{l|l|l}
\hline & \multicolumn{1}{c}{ Opções } & \multicolumn{1}{c}{$\begin{array}{c}\text { Práticas } \\
\text { Adotadas }\end{array}$} \\
\hline \multirow{3}{*}{ Abastecimento } & $\begin{array}{l}\text { a- seleção de matérias-primas } \\
\text { b- fornecedores que respeitem o meio ambiente } \\
\text { c- tecnologia verde } \\
\text { d- embalagens reutilizáveis com fornecedores e/ou clientes } \\
\text { e- redução de geração de poluição, emissões e/ou de resíduos } \\
\text { f- logística reversa }\end{array}$ & a, b, d, e, f \\
Produção & $\begin{array}{l}\text { a- tecnologia verde } \\
\text { b- redução de geração de poluição, emissões e/ ou de resíduos } \\
\text { c- ecologia industrial }\end{array}$ & b, d
\end{tabular}




\section{a- tecnologia verde}

b- embalagens reutilizáveis com fornecedores e/ou clientes

Distribuição

c- redução de geração de poluição, emissões e/ ou de resíduos

$\mathrm{b}, \mathrm{c}, \mathrm{e}$

d- ecologia industrial

e- logística reversa

Tecnologia da

a- trabalho a distância e fornecimento de tecnologias para realizá-lo

b- servidores e outros equipamentos ou computadores

c- incentivo ao uso de meios eletrônicos ao invés do papel

Informação

a- práticas de gestão de eco-design

Desenvolvimento de

Produtos

a- sistema produto-serviço

Não aplica

Geral

Fonte: Pesquisa de campo (2013)

As PME estudadas demonstram uma tendência em adotar práticas sustentáveis que decorrem de legislações, confirmando a sondagem feita pelo Sebrae com empresários do segmento, visando o debate na Conferência das Nações Unidas sobre Desenvolvimento Sustentável, no ano de 2012 (SEBRAE, 2012). Ainda que, inicialmente foca-se nisso como um requisito mínimo para funcionamento, mas as indústrias tendem a aumentar estas práticas com medidas simples e que geram resultados satisfatórios como, por exemplo, o incentivo ao uso de meios eletrônicos no lugar de papel. Muito se falou de pequenas ações para transformar o meio empresarial.

As seguir, apresenta-se o resultado do estudo quanto a percepção dos entrevistados sobre o impacto na performance ambiental e econômica, uma vez implementado as práticas sustentáveis.

Tabela 16 - Percepção dos entrevistados sobre o impacto da implementação das práticas.

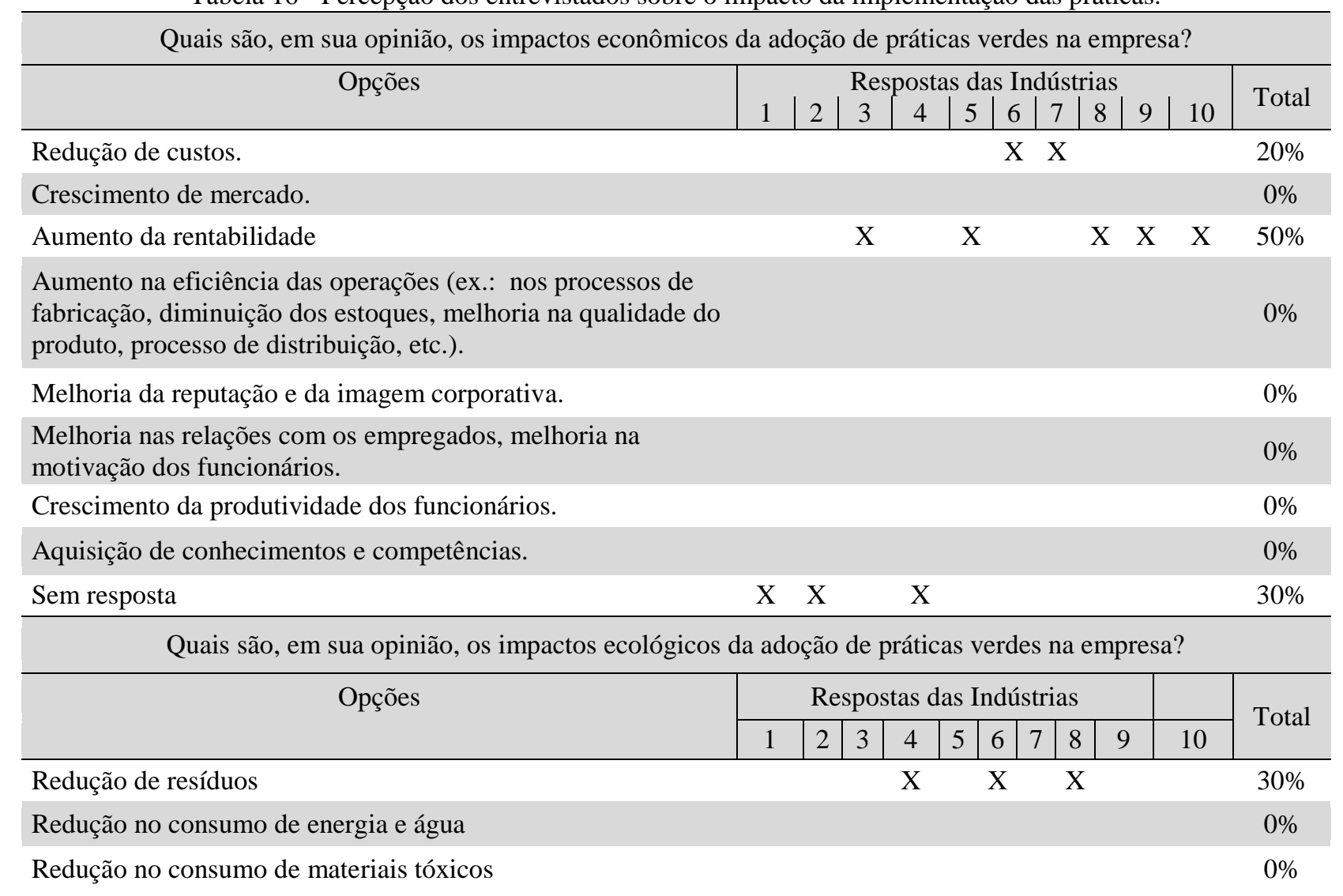




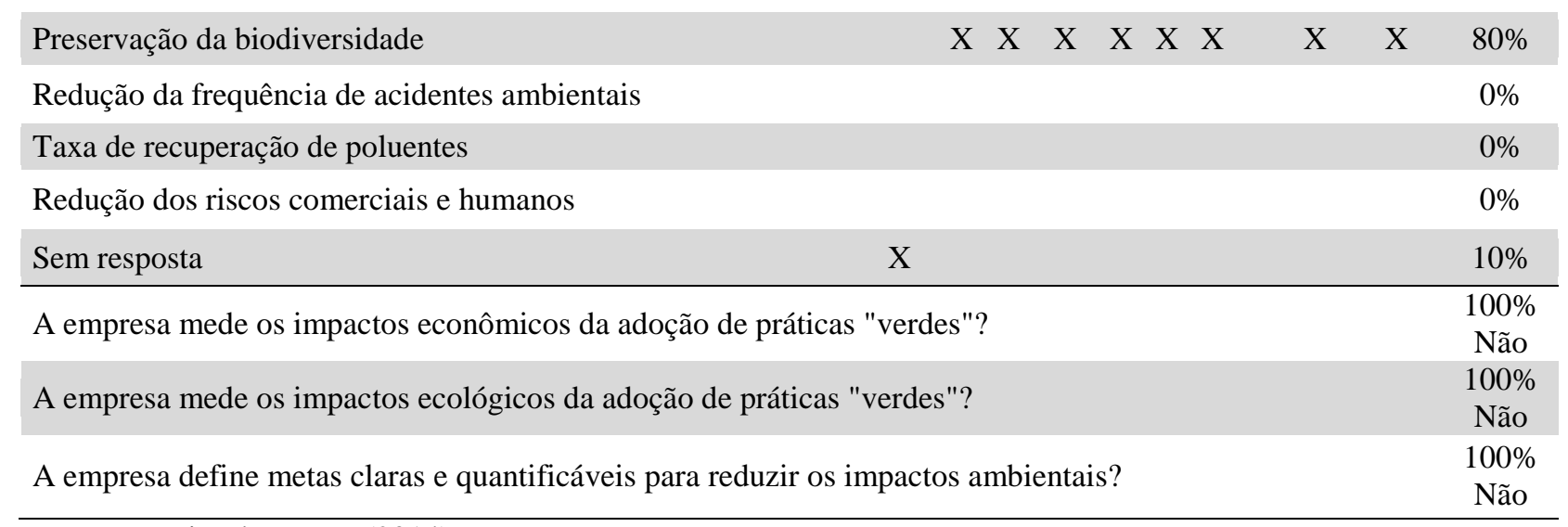

Fonte: Pesquisa de campo (2014)

Conforme a percepção dos gestores, ainda que eles não avaliem explicitamente tais impactos por meio de indicadores de performance concretos, acredita-se que as práticas de sustentabilidade podem proporcionar a redução de custos $(20 \%)$, consequentemente o aumento da rentabilidade (50\%) para as indústrias. Considere-se que o impacto econômico é positivo para aqueles que praticam sustentabilidade, apesar de os entrevistados não mencionarem elementos como, crescimento de mercado, aumento na eficiência das operações, melhoria da reputação e da imagem corporativa, melhoria nas relações com os empregados, melhoria na motivação dos funcionários, crescimento da produtividade dos funcionários ou ainda aquisição de conhecimentos e competências.

O impacto ambiental também pode ser positivo, conforme opinião dos gestores, sendo que a redução de resíduos no meio ambiente e a preservação da biodiversidade foram fatores frequentemente mencionados. Houve uma evolução natural das exigências legais de sustentabilidade, sobre a redução de geração de resíduos, que passou a fazer parte das obrigações das indústrias interessadas na certificação e qualidade ambiental, esta busca pela Ecoeficiência passa por toda a cadeia de fornecedores racionalizando o uso dos recursos naturais e diminuindo o descarte de resíduos (SISINNO et al, 2011). Apesar de o impacto na performance ambiental ser considerado positivo, não se mencionou redução no consumo de energia e água, redução no consumo de materiais tóxicos, redução da frequência de acidentes ambientais, aumento da taxa de recuperação de poluentes, ou redução dos riscos comerciais e humanos.

Finalmente, a Tabela 17 indica opinião dos gestores em relação às barreiras e aos facilitadores que impulsionam ou não às práticas de sustentabilidade.

Tabela 17 - Opinião dos gestores quanto às barreiras e os facilitadores.

\begin{tabular}{lcc}
\hline \multicolumn{1}{c|}{ Questões } & \multicolumn{2}{c}{ Respostas das Indústrias } \\
\hline $\begin{array}{l}\text { Quais são, em sua opinião, os facilitadores (ou condições } \\
\text { favoráveis) de implantação das práticas "verdes" na } \\
\text { empresa? }\end{array}$ & $\begin{array}{c}40 \% \\
\text { Fornecedores }\end{array}$ & $\begin{array}{c}\text { Políticas Públicas de } \\
\text { incentivo à PME's }\end{array}$
\end{tabular}


Quais são, em sua opinião, as barreiras de implantação das práticas "verdes" na empresa?

Fonte: Pesquisa de campo (2014) acessível
$60 \%$

Falta de incentivo por parte dos Governos

$\mathrm{Na}$ questão que aponta quem facilitaria o aumento das práticas de sustentabilidade dentro das indústrias, $40 \%$ dos entrevistados disse que depende dos fornecedores oferecerem matériaprima sustentável com preço acessível. Segundo os gestores, esse tipo de matéria-prima pode sair mais cara e, em alguns casos, dependerá de certificações para utilização, o que gera custo.

Mais que os fornecedores como facilitadores ou fonte de obstáculos, o mais citado pelos entrevistados (com cerca de $60 \%$ das respostas) são as três esferas do Governo, Municipal, Estadual e Federal. Notou-se, claramente, pelas respostas dos entrevistados, que há falta de incentivos públicos que impulsionem a continuidade e aumento da adoção das práticas de sustentabilidade.

\section{Conclusão}

Este artigo apresenta um estudo sobre as práticas de gestão sustentável na cadeia de valor em PMEs do setor químico de Uberaba, em Minas Gerais. Para tanto, por meio da metodologia de estudo de casos múltiplos, analisa-se quais são as principais motivações de adoção de práticas, quais são as práticas sendo adotadas e sua relação com o impacto percebido no desempenho ambiental e econômico das empresas estudadas. Ao todo, abordou-se mais de $60 \%$ das empresas deste setor na região.

Os resultados deste estudo indicam que quase todas as empresas estudadas possuem alguma iniciativa de gestão sustentável, mesmo que algumas delas sejam simples e de escopo limitado ao tratamento de resíduos. Isso se dá graças à motivação dos líderes das empresas, a facilidade de uso de algumas tecnologias verdes disponíveis no mercado, à visibilidade que estas mudanças trazem, e a pressão do governo (principalmente por via de legislação). Para algumas delas, a disponibilidade de matéria-prima ambientalmente corretas foi também um incentivo importante.

Além disso, pôde-se constatar que a maior parte das iniciativas verdes estão associadas à produção, com foco no tratamento de resíduos. No entanto, as empresas mencionaram frequentemente a preferência por compras de matérias primas com menores impactos ambientais, bem como a adoção de pequenas práticas em relação ao uso de Tecnologia da Informação que facilitam a gestão sustentável.

Com relação ao impacto da adoção das práticas, muitas empresas mencionaram o aumento a rentabilidade do negócio, a redução de custos, a diminuição de resíduos e do impacto na biodiversidade. No entanto, nenhuma empresa mede essas variáveis de forma explícita, e não se mencionou outros possíveis ganhos, como melhoria da reputação e da imagem corporativa, melhoria nas relações com os empregados, crescimento da produtividade, redução no consumo de 
energia e água e redução da frequência de acidentes ambientais, dentre outros. Percebe-se que a amplitude da adoção de práticas verdes talvez não seja bem avaliada ou compreendida pelas empresas estudadas.

Pôde-se notar que as empresas possuem um certo grau de preocupação com o futuro do meio ambiente e buscam, ainda que de forma simples, aplicar atitudes que favoreçam a melhoria do ecossistema. Mesmo com a falta de incentivos governamentais e com a carência de recursos humanos e financeiros, as PMEs buscam inovar na sobrevivência do mercado, criando estratégias positivas e ampliando as condições de uma possível adoção de práticas sustentáveis em sua cadeia de valor como um todo.

E mais importante, o estudo evidencia a relação positiva da adoção de práticas sustentáveis com os desempenhos econômico e ambiental das empresas estudadas, o que pode ser um elemento de incentivo para que a adoção aumente paras as PMEs, apesar de todas as restrições econômicas que essas empresas possuem.

Visando dar continuidade à tal estudo exploratório, prevê-se a realização de mais três etapas, sendo que a terceira será de cunho confirmatório, a saber: i) a ampliação do estudo para outros estados, especialmente São Paulo e Paraná; ii) a comparação com dados sendo presentemente coletados em um país desenvolvido (Canadá) e um outro emergente (Tunísia); e iii) a realização de uma survey em escala internacional sobre o tema. Futuros resultados deste estudo deverão ser apresentados nos próximos anos.

\begin{abstract}
Environmental sustainability is a topic that is now part of the agenda of many modern corporate entities. As part of this phenomenon, recent research has shown that the approach known as Green Value Chain Management has the potential to contribute to reducing companies' environmental footprint. However, most empirical studies deal with large companies, and little is known about how Small and Medium Enterprises (SMEs) have adopted such practices. Moreover, there is no indication of how SMEs of "Triangulo Mineiro", particularly chemical companies of the Uberaba region, incorporate recent sustainable management practices. This region has a major industrial park consisting of several chemical companies, which are commonly associated with environmental degradation. Thus, the objective of this work is to analyze, through a series of ten cases studies, the adoption process of sustainable practices in SMEs in the chemical sector of Uberaba region. In order to do so, we investigated their motivations, the types of practices adopted and the potential economic and environmental impacts perceived by managers. Results indicate a positive link between the adoption of such practices with economic and environmental performance of the companies studied. Furthermore, it was identified that SMEs adopt some practices mainly due to personal motivation of entrepreneurs and corporate leaders. On the other hand, the existence of limited support from government can be regarded as a factor impacting negatively on the adoption of such practices.
\end{abstract}

Key-words: green value chain management, chemical industry, environmental sustainability. 


\section{Referências}

ASSOCIAÇÃO BRASILEIRA DE INDÚSTRIA QUÍMICA. A Indústria química brasileira. São Paulo, [2013]. Disponível em: <http://www.abiquim.org.br/pdf/indQuimica/AIndustriaQuimica-SobreSetor.pdf〉. Acesso em: 5 fev. 2013.

BRASIL. Lei n ${ }^{\circ} 9.841$ de 5 de outubro de 1999. Institui o Estatuto da Microempresa e da Empresa de Pequeno Porte, dispondo sobre o tratamento jurídico diferenciado, simplificado e favorecido previsto nos arts. 170 e 179 da Constituição Federal. Diário Oficial da União, Brasília, DF, 6 out. 1999. Disponível em:

<http://www2.camara.leg.br/legin/fed/lei/1999/lei-9841-5-outubro-1999-369202-publicacaooriginal-1-pl.html_, Acessado em: 18 jun. 2012.

BRASIL. Ministério do Planejamento, Orçamento e Gestão. Resolução CONCLA nº 1, de 4 de setembro de 2006. Divulga a Classificação Nacional de Atividades Econômicas - CNAE 2.0. Diário Oficial da União, Brasília, 5 set. 2006. Disponível em: <http://www.legisweb.com.br/legislacao/?id=105027>. Acesso em: 18 jul. 2012.

BURKE, S. AND GAUGHRAN, W.F. (2006). Intelligent environmental management for SMEs in manufacturing. Robotics and Computer-Integrated Manufacturing, v. 22, n. 5-6, p. 566-575, 2006. crossref

CHIE, M.K. SHIH, L.H. An empirical study of the implementation of green supply chain management practices in the electrical and electronic industry and their relation to organizational performances. Int. J. Environ. Sci. Tech., v. 4, n. 3, p. 383-394, 2007.

FUNDAÇÃO JOÃO PINHEIRO. Resultados da amostra Censo 2010. Disponível em:< http://www.fjp.mg.gov.br/index.php/analises-demograficas/populacao-de-minas-gerais-censo-de-2010>. Acesso em: 21 out 2013.

GROZNIK, A.; ERJAVEC, J. Environmental impact of supply chains. In: GROZNIK, Ales; XIONG, Yu. Pathways to supply chain excellence. Slovenia: InTech,. 2012. cap. 7, p. 115-122. Disponível em: < http://www.intechopen.com/download/get/type/pdfs/id/32381>. Acesso em: 06 fev. 2013.

HONG, P.; KWON, H.IB. ROH, J. J. Implementation of strategic green orientation in Supply chain: An empirical study of manufacturing firms. European Journal of Innovation Management,v.12, n. 4, p. 512\532. 2009.

LEE, S.Y. Drivers for the participation of small and mediumlsized suppliers in green Supply Chain initiatives. Supply Chain Management: An International Journal, v. 13, n. 3, p.185-198. 2008. crossref

MATOS, A. C. et al. Abri minha empresa, e agora? São Paulo: SEBRAE, 2005.

NIKBAKHSH, E. Green supply chain management. In: R. Zanjirani Farahani et al., Supply Chain and Logistics in National, International and Governmental Environment. Contributions to Management Science, 2009.

PRUD'HOMME, B. La vulnérabilité des TPE et des PME dans un environnement mondialisé, 11es Journées scientifiques du Réseau Entrepreneuriat, 27, 28 et 29 mai 2009, INRPME, Trois-Rivières, Canada.

PwC, CDP Global 500 Climate Report: Sector insights: what is driving climate change action in the world's largest companies? White paper, PwC and CDP (2013).

RAO, P. HOLT, D. Do green supply chains lead to competitiveness and economic performance? International Journal of Operations \& Production Management, v. 25, n. 9, p. 8981916, 2005. crossref

RAO, P., SINGH, A. K., O’CASTILlO, O., INTALJr.,P.S.; SAJID,A. A Metric for Corporate Environmental Indicators ....for Small and Medium Enterprises in the Philippines. Business Strategy and the Environment, v. 18, p. 14-31. 2009.

RAO, P. Greening the supply chain: a new initiative in South East Asia. International Journal of Operations \& Production Management, v. 22, n.6, p. 632\655, 2002.

ROGERS, E. M. Diffusion of innovation. 5. ed. New York: The Free Press, 2003.

ROGERS, E. M. Diffusion of Innovations. New York: Free Press. 1983. 
SANTA-EULALIA, L.A.; REKIK, L.; BERGERON, F.; RETAMAL, L.; NOËL, M. Green supply chain management practices in SMEs in Québec. In: 2011 SMEs: Moving Toward Sustainable Development Conference, Network for Business Sustainability, Outubro 20-22, Montréal, Canadá, 2011.

SEBRAE MiNAS GERAIS. Anuário Estatístico Micro e Pequena Empresa. Núcleo Observatório da MPE. Belo Horizonte: SEBRAE/MG, 2009. 104 p.: il.

SEBRAE MINAS GERAIS. Sebrae em São Paulo reforça agenda de sustentabilidade. Disponível em: <www.sebrae.com.br>. Acesso em: 16 out. 2012.

SEURING, S.; SARKIS, J.; MULLER, M.; RAO, P. Sustainability and supply chain management : an introduction to the special issue. Journal of Cleaner Production, Amsterdam, v. 16, n. 15, p.1545-1551, Out. 2008.

SILVA, E.L.; MENESES, E.M. Metodologia da Pesquisa e Elaboração de Dissertação: 4a edição revisada e atualizada. Florianópolis: UFSC, 2005. 138p.

SISINNO, C. L. S.; RIZZO, A. C. L.; SANTOS, R. L. C. Ecoeficiência aplicada à redução da geração de resíduos sólidos. Rio de Janeiro: CETEM/MCT, 2011. 29 p.

SLEZYNGER, H; FIGUEIREDO, F. A trajetória da indústria química rumo à sustentabilidade. Confederação Nacional da Indústria. Associação Brasileira da Indústria Química. - Brasília: CNI, 2012. 86 p.

TAMLYN, A. The need for eco-efficiency resource tools for small and medium enterprises in Canada. Research Report, Eco-Efficiency Centre, Faculty of Management, Dalhousie University, Canada, 2007.

TAMRI, L. Adoption d'innovations environnementales dans les petites entreprises: Um modele d'analyse. 9ème CIFEPMELL'entrepreneur et la PME, vecteurs de changement et d'innovation, $28 \backslash 30$ octobre, Louvain lalNeuve, Belgium, 2008.

UBERABA (MG). Prefeitura. Uberaba: empreendedorismo e qualidade de vida num só lugar. Uberaba, [201-]. Disponível em < http://www.uberaba.mg.gov.br/portal/conteudo,615>. Acesso em: 25 out. 2013.

WONGTSCHOWSKI, P. A indústria química brasileira - desafios e oportunidades. Journal of the Brazilian Chemical Society, São Paulo, v. 22, n. 4, p. 605-608, 2011. crossref

WONGTSCHOWSKI, P. Indústria química. Ciência Hoje, São Paulo, v. 47, p. 36, abr. 2011.

YIN, R., 2009. Case Study Research: Design and Methods. Sage, United States of America.

ZHU, Q., SARKIS, J. GENG, Y. Green supply chain management in China: pressures, practices and performance. International Journal of Operations \& Production Management, v. 25, n. 5, p. 449-468, 2005. crossref

\section{Dados dos autores:}

\section{Nome completo: Andréia Fernandes Malaquias}

Filiação institucional: Universidade Federal do Triângulo Mineiro

Departamento: Instituto de Ciências Exatas, Naturais e Educação

Função ou cargo ocupado: Assistente em Administração

Endereço completo para correspondência (bairro, cidade, estado, país e CEP): Rua Guia Lopes,

1131, Abadia, Uberaba, Minas Gerais, Brasil, 38026-360

Telefones para contato: 34 3318-5582 - 3315-2310

e-mail: andreiafmalaquias@yahoo.com.br 
Nome completo: Luis Antonio De Santa-Eulalia

Filiação institucional: Université de Sherbrooke

Departamento: Faculté d'administration

Função ou cargo ocupado: Professor Adjunto

Endereço completo para correspondência (bairro, cidade, estado, país e CEP): bureau K1-4012, 2500, boul. de l'Université, Sherbrooke (Québec), CANADA, J1K 2R1

Telefones para contato: $+1(819) 821-8000$, ramal 65042

e-mail:1.santa-eulalia@usherbrooke.ca

Nome completo: Rui Tadashi Yoshino

Filiação institucional: Universidade Tecnológica Federal do Paraná

Departamento: Engenharia de Produção

Função ou cargo ocupado: Professor Adjunto

Endereço completo para correspondência (bairro, cidade, estado, país e CEP): Av. Monteiro

Lobato s/n km 04 (042) 32204885 Telefones para contato: 34 3318-5600

e-mail: ruiyoshino@utfpr.edu.br

Nome completo: David Calchau Jorge

Filiação institucional: Universidade Federal do Triângulo Mineiro

Departamento: Engenharia Elétrica

Função ou cargo ocupado: Professor Adjunto

Endereço completo para correspondência (bairro, cidade, estado, país e CEP): Av. Dr. Randolfo

Borges Jr., 1.250, Univerdecidade, Uberaba, Minas Gerais, Brasil, 38064-200

Telefones para contato: 34 3318-5600

e-mail:david@eletrica.uftm.edu.br

Submetido em: 23/03/2014

Aceito em: 17/12/2014 